\title{
Entropy Generation of MHD Poiseuille Flow with Hall and Joule Heating Effects
}

\author{
A. A. Opanuga*, O. O. Agboola, H. I. Okagbue, A. M. Olanrewaju
}

\begin{abstract}
In this article investigation has been conducted on the effects of Hall parameter, rotation parameter and Joule heating on the entropy generation of fully developed electrically conducting Poiseuille flow. The coupled system of ordinary differential equations for the flow are obtained, non-dimensionalised and solutions are constructed by Adomian decomposition technique. The effects of Hall current, Ion-slip, Joule heating and magnetic parameters on the velocity, temperature, entropy generation and Bejan number are explained and shown graphically. The results indicate that fluid entropy generation is induced by increase in Hall current, rotation and Joule heating parameters. Furthermore Bejan number is accelerated by Hall current, rotation, Magnetic and Joule heating parameters which signifies that heat transfer irreversibility dominates entropy generation.
\end{abstract}

Keywords - Poiseuille flow, Hall current, Ion-slip, Entropy generation, Adomian decomposition method (ADM).

\section{INTRODUCTION}

Tnvestigation regarding Magnetohydrodynamic flows was 1 pioneered by the discoveries of Alfven and others in the 1940s [1]. Magnetohydrodynamics (MHD) has been described as the study of the interplay between magnetic fields and conducting fluids. Such fluids must be electrically conducting; these include liquid metals (such as mercury, gallium, molten magnesium, molten antimony, liquid sodium etc.), plasmas (ionized gases or electrically conducting gases) such as solar atmosphere and salt water or electrolyte. Since its introduction in the past half century several engineering and industrial problems have been addressed: lubrication control of highspeed spinning machine components with magnetic fields, improved MHD generators [2], crystal growth control [3], magnetoastronautical flows [4], fusion reaction [5], electrolysis (reduction of aluminium oxide to aluminium) [6]. Due to the significance of MHD flows appreciable studies have been reported in literature, MHD Couette flow [7-9], MHD Poiseuille flow [10-11], MHD couple stress fluid [1214], MHD nanofluid [15-17], MHD second grade fluid [1820], MHD third grade fluid [21-23], MHD micropolar fluid [24-25], other interesting investigations are in refs.[26-33]. In

This work was supported Covenant University Centre for Research Innovation and Discovery (CUCRID)

A.A. Opanuga, O.O. Agboola, H.I. Okagbue and A.M. Olanrewaju are with the Department of Mathematics, Covenant University, Ota, Nigeria (e-mail: A. A. Opanuga: abiodun.opanuga@covenantuniversity.edu.ng, O. O. Agboola: ola.agboola@covenantuniversity.edu.ng, H. I. Okagbue: hilary.okagbue@covenantuniversity.edu.ng, A. M. Olanrewaju: anuoluwapo.olanrewaju@covenantuniversity.edu.ng). addition, several investigations have been conducted to incorporate Hall-magnetohydrodynamics, rotating magnetohydrodynamic flows in the study of magnetohydrodynamic.

In all the previously mentioned investigations on magnetohydrodynamics, an assumption of small and moderate magnetic field was taken in the application of Ohm's law resulting in unnoticeable impact in the flows. However current trend in the application of MHD is towards strong magnetic field owing to its numerous applications in magnetic fusion systems, electrically-conducting aerodynamics, energy generators, Hall accelerators and flight magnetohydrodynamics. Lighthill [34] was the first to suggest the inclusion of Hall effect in MHD flows, notwithstanding Sato [35] conducted the first significant research of Hall current effects on magnetohydrodynamic boundary layers, it was reported that such flow becomes secondary in nature. Furthermore, investigations have revealed that the interaction of the Coriolis and electromagnetic forces cannot be ignored in MHD flows. It is noteworthy that Coriolis and MHD forces are comparable in magnitude and Coriolis force stimulates secondary flow in the fluid. Rotating magnetohydrodynamic flows have practical applications in the turbo machinery, the solidification process in metallurgy, and some astrophysical problems. In view of the foregoing several investigators have studied the significance of Hall and rotating magnetohydrodynamcs in various fluid flows under different configurations. Mohanty [36] investigated MHD flow in a rotating channel. Jana et al. [37] studied the hydromagnetic Couette flow and heat transfer with Ion-slip effect. Hall current effect on hydromagnetic Couette flow in a rotating system was considered by Jana and Datta [38]. Seth et al. [39] studied an unsteady MHD Couette flow in a rotating system. Rao and Rao [40] studied the MHD flow of Rivlin-Ericksen fluid of rotating second grade contained between two infinite, parallel plates. Kasiviswanathan and Gandhi [41] reported on MHD flow of rotating micropolar fluid between two infinite parallel, rotating disks.

In this article detailed analysis of the effects of Hall current and Ion-slip on electrically conducting Poiseuille flow in a rotating frame of reference is considered. To the best of authors' knowledge the irreversibility associated with the Hall current and Ion-slip effects of this important flow has not been adequately addressed. First law of thermodynamics approach has been applied in several investigations reported in literature, however in several engineering processes, factors responsible for exergy loss in various fluid flows are of interest 
because information regarding factors responsible for such destruction will enhance system design and energy conservation. Second law of thermodynamics is applied in this work, the approach which has developed to a new discipline being referred to as entropy generation minimization (EGM), it was pioneered by Bejan [42] and several other investigators have applied it various fluid flow; third grade fluid [43], micropolar fluid [44], couple stress fluid [45-46], Casson fluid [47], Couette flow [47] and others [48-50].

In spite of several techniques such as Homotopy perturbation method [51], differential transform method [52-53], variational iteration technique [54], finite difference technique [55], Network Simulation method [56] etc. reported in literature for the solution various fluid models, the robust technique of Adomian decomposition is employed to solve the dimensionless momentum and energy equations obtained from the model equations owing to its rapid convergence and simplicity in application as reported in the following references [57-59]. The organization of the rest of the paper is as follows: section two presents derivation of the governing equations, Adomian decomposition method of solution is treated in section three, section four is based on the discussion of results and the concluding remarks are made in section five.

\section{MATHEMATICAL ANALYSIS}

Steady, viscous and incompressible electrically conducting Poiseuille flow between infinite horizontal parallel plates with thickness $h$ is considered. A uniform magnetic field of strength $B_{0}$ is applied perpendicularly to the direction of flow, ignoring the induced magnetic fields due to the assumption of a very small magnetic Reynolds number. The $x$-axis is along the lower plate in the flow direction, the $y$-axis is perpendicular to the channel plates while the $z$-axis is normal to $x y$ - plane. Furthermore, assumption of relatively high electron-atom collision frequency is taken so that the effects of Hall current and ion slip are considered. The fluid is rotating with an angular velocity $\Omega^{*}$ about the normal to the plate as shown in Figure 1. The governing equations of flow in a rotating frame of reference under the assumptions made above are [11].

$$
\begin{aligned}
& \rho v_{\circ} \frac{d u^{*}}{d y^{*}}+2 \Omega^{*} w^{*}=\mu \frac{d^{2} u^{*}}{d y^{* 2}}-\frac{d p}{d x}-\frac{\sigma B_{0}^{2}}{1+m^{2}}\left(u^{*}-m w^{*}\right) ; \\
& u^{*}(0)=0=u^{*}(h), \\
& \rho v_{\circ} \frac{d w^{*}}{d y^{*}}-2 \Omega^{*} u^{*}=\mu \frac{d^{2} w^{*}}{d y^{* 2}}-\frac{\sigma B_{0}^{2}}{1+m^{2}}\left(w^{*}+m u^{*}\right) ; \\
& w^{*}(0)=0=w^{*}(h), \\
& \rho c_{p} \frac{d T^{*}}{d y^{*}}=k \frac{d^{2} T^{*}}{d y^{* 2}}+\mu\left[\left(\frac{d u^{*}}{d y^{*}}\right)^{2}+\left(\frac{d w^{*}}{d y^{*}}\right)^{2}\right]+ \\
& \sigma B_{0}^{2}\left(w^{* 2}+u^{* 2}\right) ; T^{*}(0)=T^{*}(h)=0 .
\end{aligned}
$$

Introducing the non-dimensional expressions into equations (1) - (3),

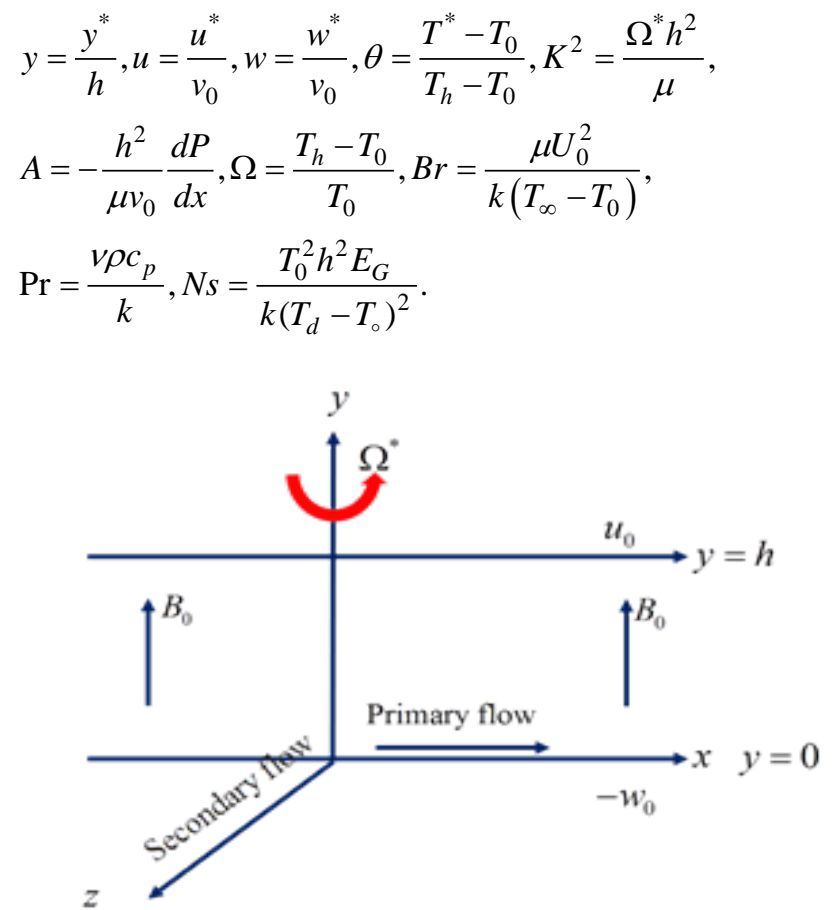

Fig.1: Physical representation of the model

The dimensionless coupled differential equations below are obtained as

$$
\begin{aligned}
& s \frac{d u}{d y}+2 K^{2} w=A+\frac{d^{2} u}{d y^{2}}-\frac{M^{2}}{1+m^{2}}(u-m w) ; u(0)=0=u(1), \\
& s \frac{d w}{d y}-2 K^{2} u=\frac{d^{2} w}{d y^{2}}-\frac{M^{2}}{1+m^{2}}(w+m u) ; w(0)=0=w(1) \\
& s p_{r} \frac{d \theta}{d y}=\frac{d^{2} \theta}{d y^{2}}+B r\left[\left(\frac{d u}{d y}\right)^{2}+\left(\frac{d w}{d y}\right)^{2}\right]+J\left(w^{2}+u^{2}\right) \\
& \theta(0)=\theta(1)=0 .
\end{aligned}
$$

In equations (1)-(7) $\left(u^{*}, u\right)$ are dimensional and dimensionless velocity components respectively along $x$-axis, $\left(w^{*}, w\right)$ are dimensional and dimensionless velocity component respectively along $z$-axis, $\left(T^{*}, \theta\right)$ are the dimensional and dimensionless fluid temperature respectively, $\left(k, K^{2}\right)$ are fluid thermal conductivity and rotation parameter respectively, $\left(\Omega, \Omega^{*}\right)$ are temperature difference and angular velocity respectively, $\left(B_{0}^{2}, M^{2}\right)$ are uniform transverse magnetic field and magnetic field parameter respectively, $\left(v_{0}, s\right)$ are constant velocity of fluid suction/injection and suction/injection parameter respectively, $\mu$ is the dynamic viscosity, $p$ is fluid pressure, $\sigma$ electrical conductivity of the fluid, $h$ is channel width, $m$ is Hall parameter, $J$ is Joule heating parameter, $\rho$ is fluid density, 
Pr is Prandtl number, $C_{p}$ is specific heat at constant pressure and $\mathrm{Br}$ is the Brinkman number.

\section{METHOD OF SOLUTION BY ADM}

By direct integration of equations (5) - (7) the following forms are obtained

$u(y)=a y+\int_{0}^{y} \int_{0}^{y}\left\{s \frac{d u}{d Y}-2 K^{2} w-A+\frac{M^{2}}{1+m^{2}}(u-m w)\right\} d Y d Y$, (8)

$w(y)=b y+\int_{0}^{y} \int_{0}^{y}\left\{s \frac{d w}{d Y}+2 K^{2} u+\frac{M^{2}}{1+m^{2}}(w+m u)\right\} d Y d Y$,

$\theta(y)=$

$f y-\int_{0}^{y} \int_{0}^{y}\left\{s p_{r} \frac{d \theta}{d y}-\left(\begin{array}{l}\left.B r\left[\left(\frac{d u}{d y}\right)^{2}+\left(\frac{d w}{d y}\right)^{2}\right]+\right) \\ J\left(u^{2}+w^{2}\right)\end{array}\right\} d Y d Y\right.$.

By Adomian decomposition method of solution it is assumed that

$u(y)=\sum_{n=0}^{\infty} u_{n}(y), w(y)=\sum_{n=0}^{\infty} w_{n}(y), \theta(y)=\sum_{n=0}^{\infty} \theta_{n}(y)$,

In view of (11) equations (8) - (10) yield the following recurrence relations

$\sum_{n=0}^{\infty} u_{0}(y)=a y-\int_{0}^{y} \int_{0}^{y}\{A\} d Y d Y ;$
$\sum_{n=0}^{\infty} u_{n+1}(y)=\int_{0}^{y} \int_{0}^{y}\left\{s \frac{d u}{d Y}+2 K^{2} w+\frac{M^{2}}{1+m^{2}}(u-m w)\right\} d Y d Y$,

$\sum_{n=0}^{\infty} w_{0}(y)=b y$;

$\sum_{n=0}^{\infty} w_{n+1}(y)=\int_{0}^{y} \int_{0}^{y}\left\{s \frac{d w}{d Y}+2 K^{2} u+\frac{M^{2}}{1+m^{2}}(w+m u)\right\} d Y d Y$,

$\sum_{n=0}^{\infty} \theta_{0}(y)=f y$

$\sum_{n=0}^{\infty} \theta_{n+1}(y)=\int_{0}^{y} \int_{0}^{y}\left\{s p_{r} \frac{d \theta}{d y}-\left(\begin{array}{l}B r\left[D_{n}+E_{n}\right]+ \\ J\left(F_{n}+H_{n}\right)\end{array}\right)\right\} d Y d Y$,

The non-linear terms in equation (14) are identified as follows

$D_{n}=\left(\frac{d u}{d y}\right)^{2}, E_{n}=\left(\frac{d w}{d y}\right)^{2}, F_{n}=u^{2}, H_{n}=w^{2}$

and the Adomian polynomials are decomposed as

$$
\left.\begin{array}{l}
D_{0}=\left(\frac{d u_{0}}{d y}\right)^{2}, D_{1}=2\left(\frac{d u_{0}}{d y}\right)^{2} \frac{d u_{1}}{d y}, \\
D_{2}=\left(\frac{d u_{1}}{d y}\right)^{2}+2 \frac{d u_{0}}{d y} \frac{d u_{2}}{d y} \\
E_{0}=\left(\frac{d w_{0}}{d y}\right)^{2}, E_{1}=2\left(\frac{d w_{0}}{d y}\right)^{2} \frac{d w_{1}}{d y}, \\
E_{2}=\left(\frac{d w_{1}}{d y}\right)^{2}+2 \frac{d w_{0}}{d y} \frac{d w_{2}}{d y} \\
F_{0}=u_{0}^{2}, F_{1}=2 u_{0} u_{1}, F_{2}=u_{0}^{2}+2 u_{0} u_{2} \\
H_{0}=w_{0}^{2}, H_{1}=2 w_{0} w_{1}, H_{2}=w_{0}^{2}+2 w_{0} w_{2}
\end{array}\right\} .
$$

The exact solution of Eq. (5) is given in Eq. (18) and this is compared with the Adomian decomposition result and presented in Table 1 to validate the result of this analysis. $u(y)=-0.026463916072631308 e^{-1.9024984394500783 y}$

$\left(\begin{array}{l}8.446825606379067- \\ 9.446825606379067 \mathrm{e}^{1.9024984394500783 y}+ \\ 1 . e^{4.004996878900157 y}\end{array}\right)$

Table 1. Comparison of Results at $K=m=0, \quad A=1$,

\begin{tabular}{|c|c|c|c|}
\hline S/N & $\sum U_{E X A C T}$ & $\sum_{y=0}^{15} U_{A D M}$ & ERROR \\
\hline 0.1 & 0.0325348622 & 0.0325348622 & $-4.0 \times 10^{-11}$ \\
\hline 0.2 & 0.0569114718 & 0.0569114718 & 0.00000 \\
\hline 0.3 & 0.0739531887 & 0.0739531887 & $3.0 \times 10^{-11}$ \\
\hline 0.4 & 0.0842026674 & 0.0842026674 & $-3.0 \times 10^{11}$ \\
\hline 0.5 & 0.0879381842 & 0.0879381842 & $1.0 \times 10^{-10}$ \\
\hline 0.6 & 0.0851795771 & 0.0851795771 & $1.0 \times 10^{-10}$ \\
\hline 0.7 & 0.0756838288 & 0.0756838288 & $3.0 \times 10^{-10}$ \\
\hline 0.8 & 0.0589299059 & 0.0589299059 & $2.0 \times 10^{-10}$ \\
\hline 0.9 & 0.03409202082 & 0.03409202082 & $1.0 \times 10^{-10}$ \\
\hline
\end{tabular}

NOTE: $E R R O R=U_{\text {EXACT }}-U_{\text {ADM }}$

The local entropy generation for the flow is given as Bejan [42]

$$
\begin{aligned}
& E_{G}=\frac{k}{T_{0}^{2}}\left(\frac{d T^{*}}{d y^{*}}\right)^{2}+\frac{\mu}{T_{0}}\left(\left(\frac{d u^{*}}{d y^{*}}\right)^{2}+\left(\frac{d w^{*}}{d y^{*}}\right)^{2}\right)+ \\
& \frac{\sigma B_{0}^{2}}{T_{0}}\left(w^{* 2}+u^{*^{2}}\right),
\end{aligned}
$$


The first term in equation (19) $\frac{k}{T_{0}^{2}}\left(\frac{d T^{*}}{d y^{*}}\right)^{2}$ is entropy generation due to heat transfer, the next term $\frac{\mu}{T_{0}}\left(\left(\frac{d u^{*}}{d y^{*}}\right)^{2}+\left(\frac{d w^{*}}{d y^{*}}\right)^{2}\right)$ is entropy generation due to viscous dissipation and $\frac{\sigma B_{0}^{2}}{T_{0}}\left(w^{*^{2}}+u^{*^{2}}\right)$ is magnetic field entropy generation.

Using Eq. (4) in Eq. (18) the dimensionless form of entropy generation is becomes

$$
N s=\left(\frac{d \theta}{d y}\right)^{2}+\frac{B r}{\Omega}\left(\left(\frac{d u}{d y}\right)^{2}+\left(\frac{d w}{d y}\right)^{2}\right)+\frac{J}{\Omega}\left(w^{2}+u^{2}\right),
$$

where $\left(E_{G}, N s\right)$ are the dimensional and dimensionless entropy generation rate.

The ratio of heat transfer entropy generation $\left(N_{1}\right)$ to fluid friction entropy generation $\left(N_{2}\right)$ is represented as

$$
\Phi=\frac{N_{2}}{N_{1}}
$$

Alternatively, Bejan number gives the entropy generation distribution ratio parameter; it represents the ratio of heat transfer entropy generation $\left(N_{1}\right)$ to the total entropy generation $\left(N_{s}\right)$ due to heat transfer and fluid friction, it is defined as

$$
\mathrm{Be}=\frac{N_{1}}{N_{s}}=\frac{1}{1+\Phi}
$$

Clearly, Eq. (22) reveals that Bejan number ranges from 0 to 1 . $B e=0$ represents the limit at which fluid friction irreversibility dominates entropy generation, while $B e=1$ corresponds to the dominance of heat transfer irreversibility over fluid friction irreversibility and $B e=0.5$ is the case when heat transfer and fluid friction entropy generation rates are equal

\section{RESUlTS AND DISCUSSION}

\section{A. Velocity Profiles}

Effects of Hall parameter on primary velocity and secondary velocity are displayed in Figures 2A and 2B. Fluid motion in both directions is enhanced as shown in the plots. This is attributed to the weakening of the magnetic resistivity by the inclusion of Hall parameter. The term $(m)$ which represents Hall current is appearing as a denominator in $\left(\frac{M^{2}}{1+m^{2}}\right)$. Therefore increasing the values of $(m)$ when $(M)$ is fixed will reduce the resistive force imposed by the magnetic parameter. In Figures $3 \mathrm{~A}$ and $3 \mathrm{~B}$ it is observed that increase in the rotation parameter $\left(K^{2}\right)$ decelerates primary velocity while on the other hand it boosts secondary velocity. This is due to the effect of Coriolis force that is dominant in the region close to the axis of rotation. It is an established fact reported in literature that rotation usually enhances secondary fluid velocity in the flow-field by retarding the primary fluid velocity.

Next is the effect of magnetic parameter on fluid motion, it is noted in Figures $4 \mathrm{~A}$ and $4 \mathrm{~B}$ that fluid velocity is considerably impeded as the values of magnetic parameter increases. This phenomenon is well established in literature by many authors. The underlying reason for such behavior is the presence of Lorentz forces as a result of the applied magnetic field which is opposite to the flow direction.

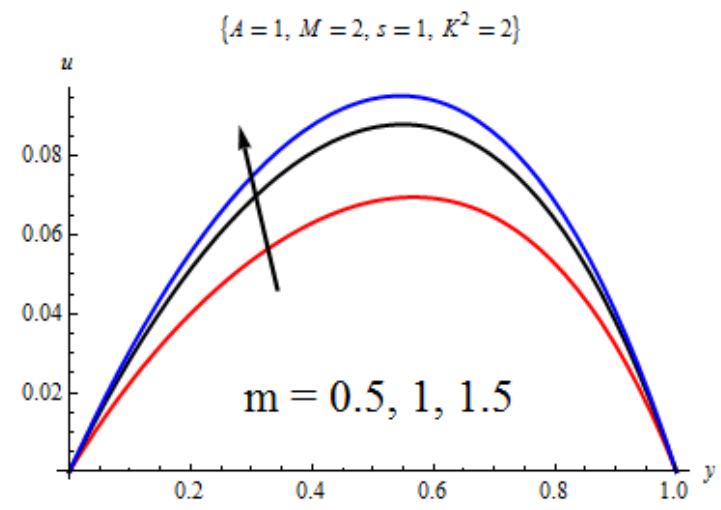

Fig.2A: Primary velocity versus Hall parameter

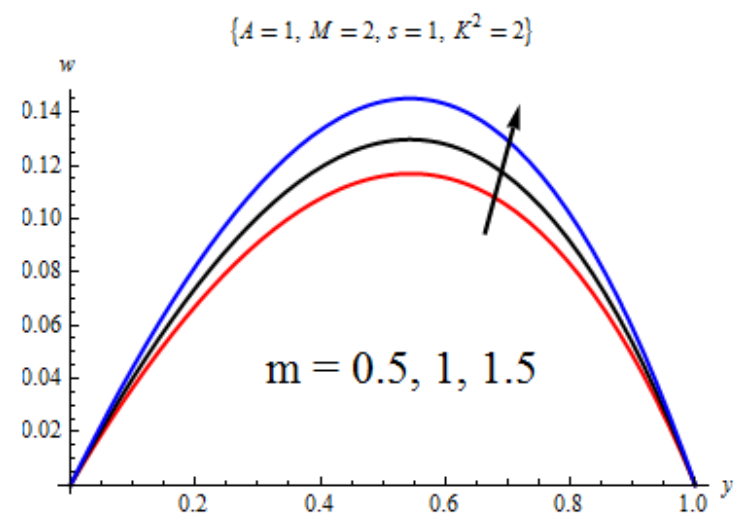

Fig.2B: Secondary velocity versus Hall parameter

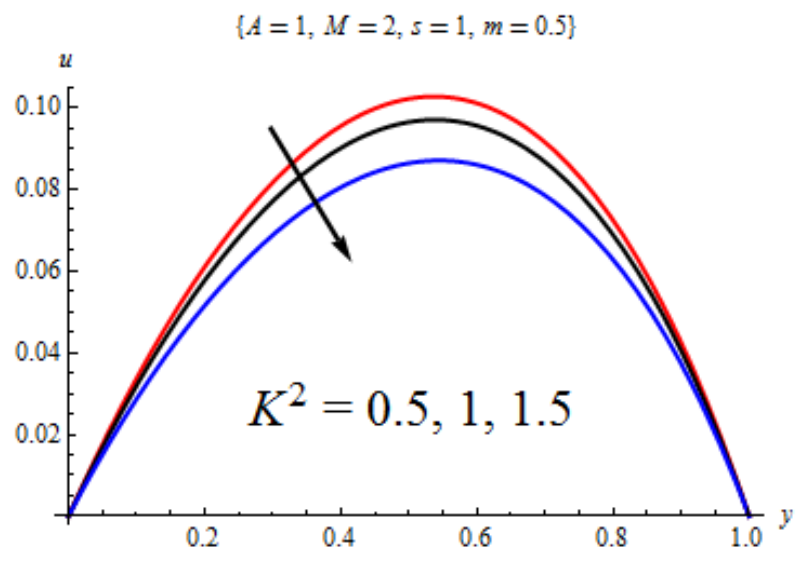

Fig.3A: Primary velocity versus rotation parameter 


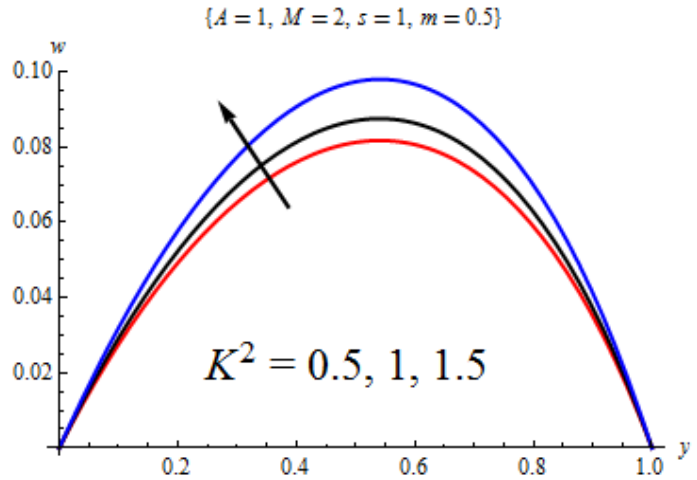

Fig.3B: Secondary velocity versus rotation parameter

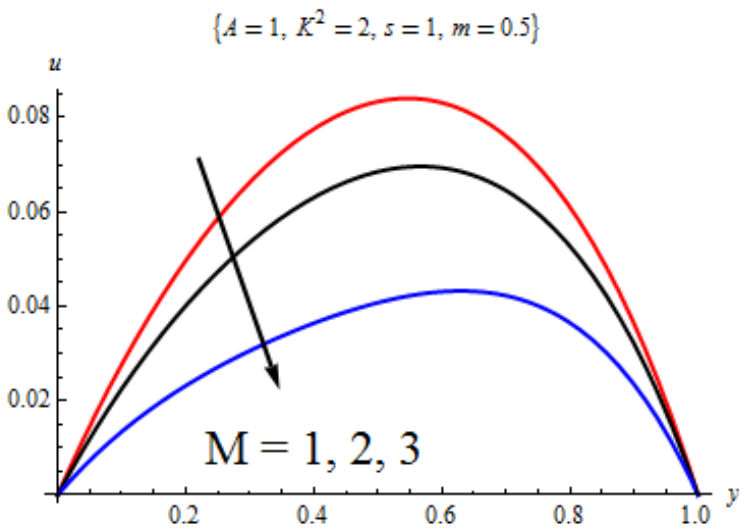

Fig.4A: Primary velocity versus magnetic parameter

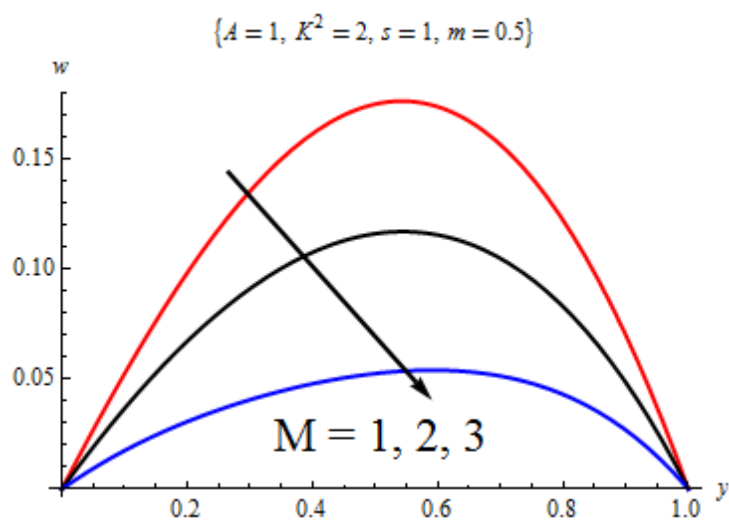

Fig.4B: Secondary velocity versus magnetic parameter

\section{B. Temperature Profiles}

Figures 5 and 6 illustrate the effects of Hall current and rotation parameters on fluid temperature, it is observed that Hall parameter does not have much significant influence on fluid temperature as depicted in Figure 5. However reduction in fluid temperature is noticed in the region $0.2 \leq y \leq 0.85$. The reduction in fluid temperature is traced to the fact that inclusion of Hall parameter in the Navier-Stoke equation reduces the fluid magnetic resistivity force as displayed in Figure 2. In Figure 6 it is observed that increase in rotation parameter does not have any remarkable effect on fluid temperature. Figures 7 and 8 indicate that a rise in the values of Joule heating and magnetic parameters result to a corresponding enhancement in fluid temperature. The rise in fluid temperature is connected to the term that represents Joule heating parameter in the energy equation; it is the product of magnetic parameter and Brinkman number, and increase in Brinkman number enhances fluid viscous dissipation. In addition, viscous heating becomes more pronounced due to the Lorentz heating effect from the magnetic parameter. The collective effect of all these is the significant rise in fluid temperature as illustrated in Figures 7 and 8.

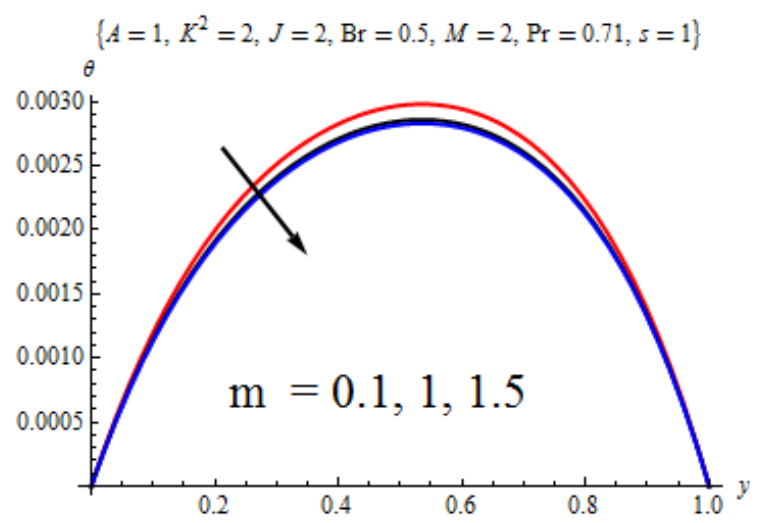

Fig.5: Temperature versus Hall parameter

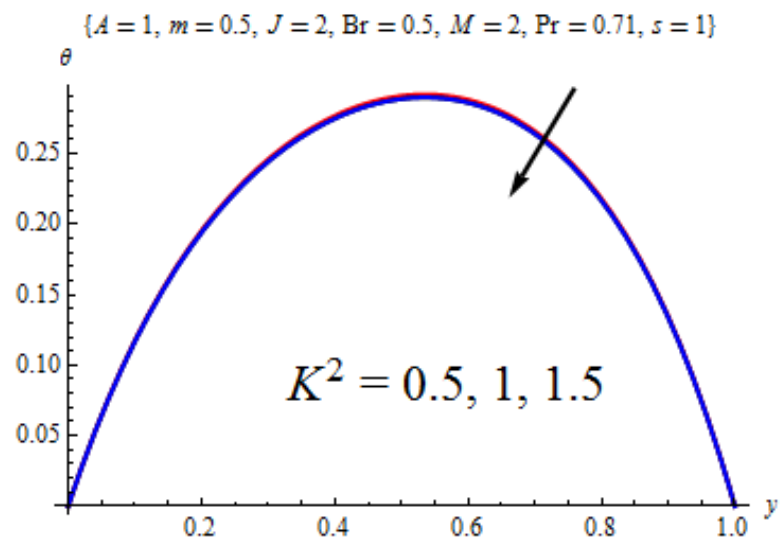

Fig.6: Temperature profile versus rotation parameter

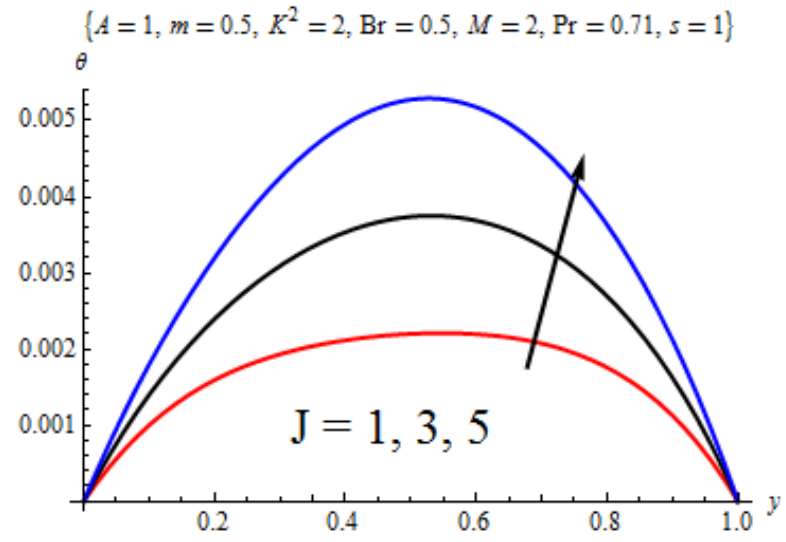

Fig.7: Temperature profile versus Joule heating parameter 


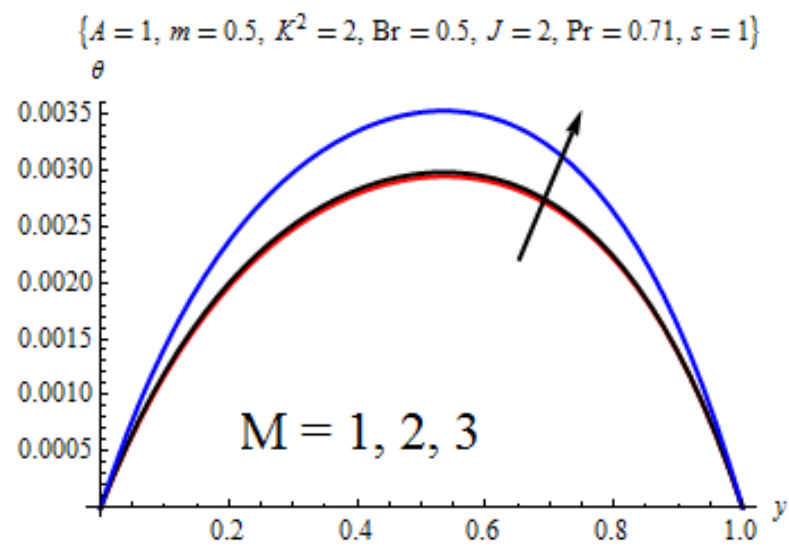

Fig.8: Temperature profile versus magnetic parameter

\section{Entropy Generation}

Effects of Hall parameter, rotation parameter and Joule heating parameter on entropy generation rate are shown in Figures 9, 10 and 11 . Generally entropy generation is enhanced as the parameters take higher values. As depicted in Figures 2A and $2 \mathrm{~B}$, Hall parameter raises fluid motion by supressing the magnetic resistive force, the net effect is the increased entropy production as shown in Figure 9. Similar explanation holds for the effect of rotation parameter on fluid irreversibility, as portrayed in Figure 10 entropy generation received a boost as rotation parameter values increase. In Figure 11 it is noteworthy to observe that entropy generation is slightly raised at the centreline of the channel but the effect is not significant at the channel walls as Joule heating parameter increases.

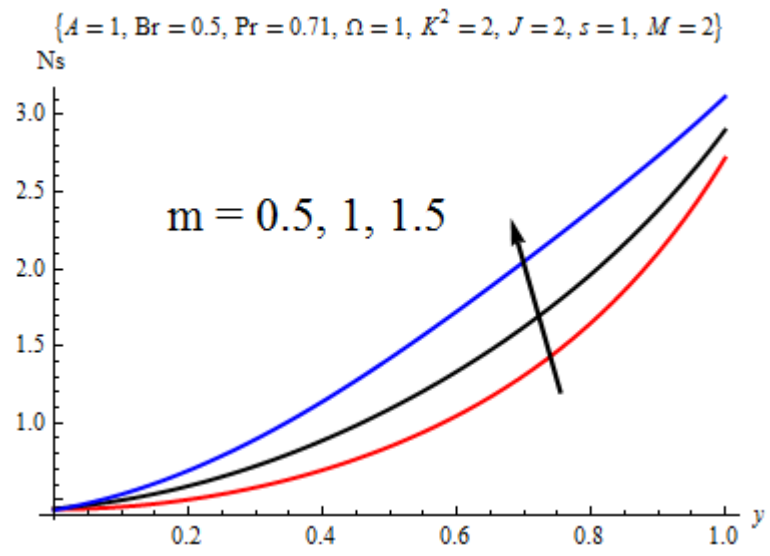

Fig.9: Entropy generation versus Hall parameter

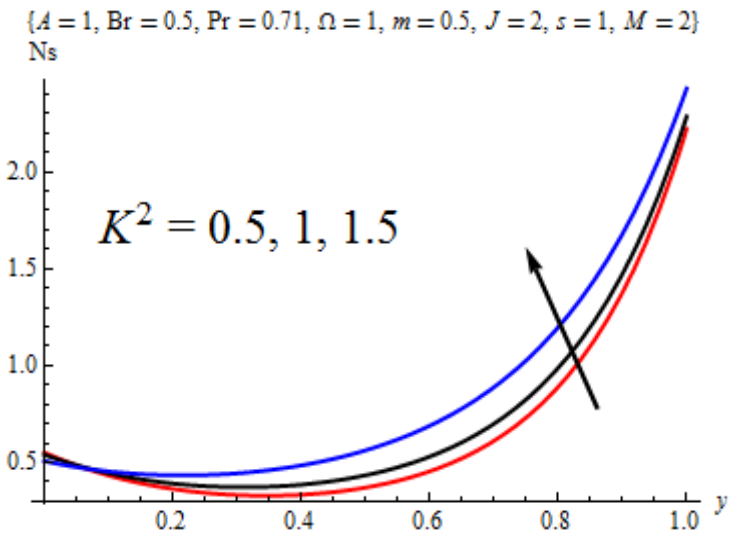

Fig.10: Entropy generation versus rotation parameter

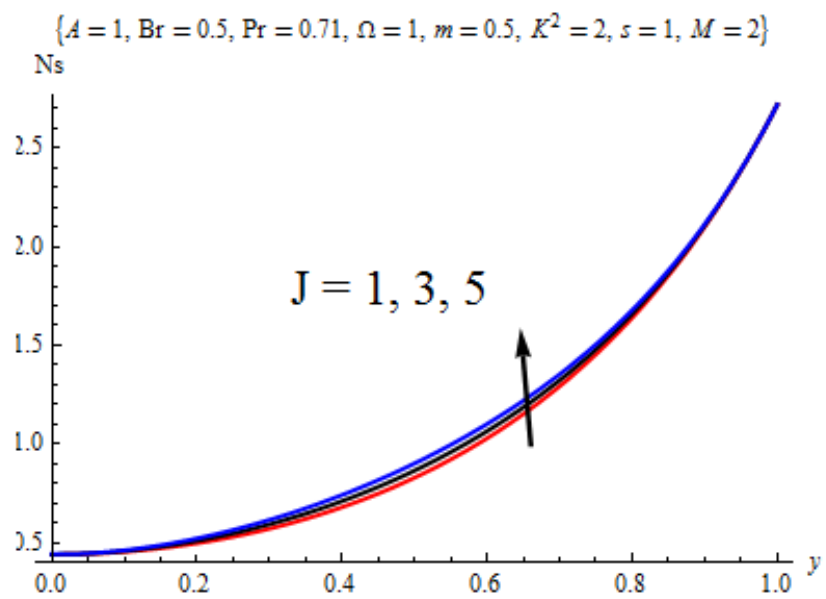

Fig.11: Entropy generation versus Joule heating parameter

D. Irreversibility Ratio

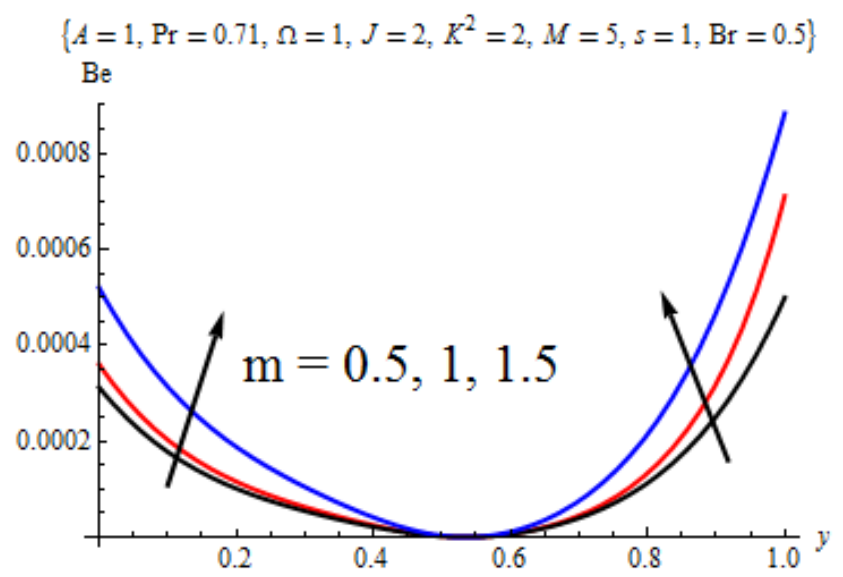

Fig.12: Bejan number versus Hall parameter 
Figures 12 to 15 describe the influence of Hall parameter, rotation parameter, Joule heating parameter and magnetic parameter on fluid irreversibility ratio. Apparently Bejan number is enhanced as each of the parameter increases. This is an indication that heat transfer irreversibility dominates entropy generation.

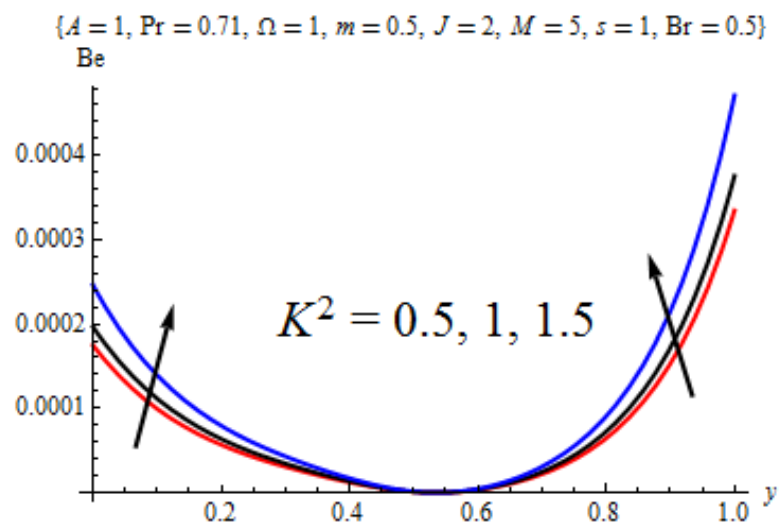

Fig.13: Bejan number versus rotation parameter

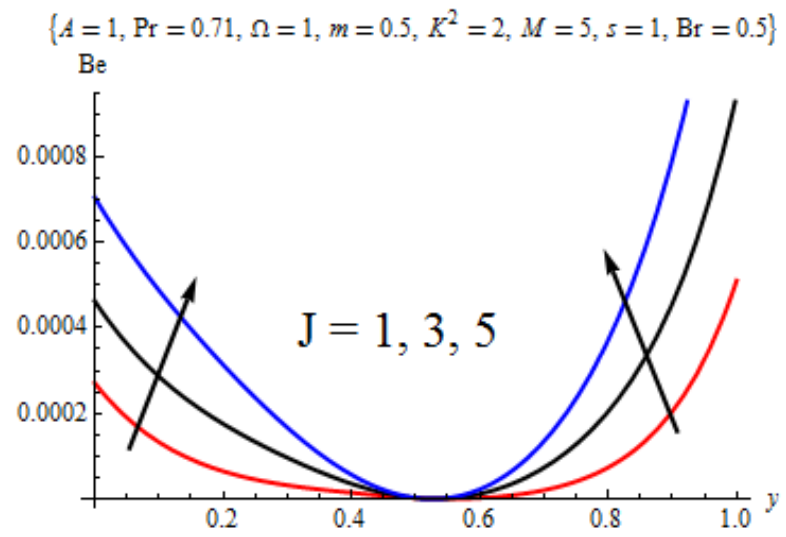

Fig.14: Bejan number versus Joule parameter

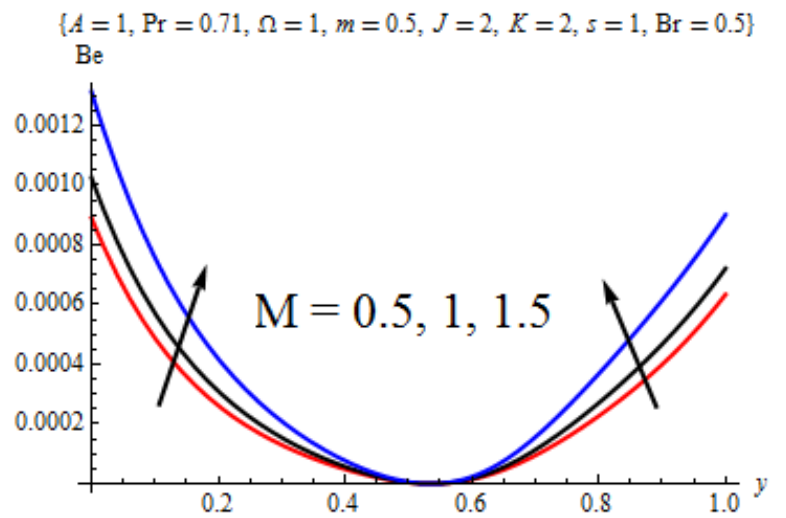

Fig.15: Bejan number versus magnetic parameter

\section{CONCLUSION}

An analytical investigation of Hall current and Joule heating effects on entropy generation of rotating Poiseuille flow has been conducted. The obtained governing equations are nondimensionalised and solved using Adomian decomposition technique. The solution is used to determine entropy generation rate and irreversibility ratio. Plots are presented to explain the physics of the flow.

Flow with stationary channel plates maintained at constant and equal temperatures is referred to as Poisuille flow. Such flow has applications in the following areas; blood flows through the arteries and human heart, motion of fluid in car engine and the control of fluid speed by the application of Lorentz force in metallurgical industry. In addition, the discovery that heat transfer irreversibility dominates entropy production would improve entropy generation minimization in system design and manufacturing industries. This paper is limited to heat transfer Poiseuille flow, future research can include mass transfer and thermal buoyancy

From the results it is concluded that:

1. Hall parameter enhances both primary and secondary velocity,

2. Rotation parameter increases secondary velocity but decreases primary velocity,

3. Magnetic parameter decreases both primary and secondary velocity,

4. Fluid temperature is reduced by both Hall current and rotation parameters while Joule and magnetic parameter boost it,

5. Hall current, rotation and Joule heating parameters induce entropy production,

6. Bejan number rises due to increase in Hall current, rotation, magnetic and Joule heating parameters, signifying the dominance of heat transfer irreversibility.

\section{REFERENCES}

[1] H. Alfven, "Cosmical electrodynamics". Oxford university press, reprint, 1953

[2] C. Vogin, A. Alemany, "Analysis of the flow in a thermo-acoustic MHD generator with conducting walls”. Eur J Mech B. 2007, vol. 26, no. 4, pp. 479-493

[3] H. Yasuda, "Applications of high magnetic fields in materials processing” In: Molokov, S., Moreau, R. and Moffatt, K. Magnetohydrodynamics: historical evolution and trends. Fluid mechanics and its applications, 80, Part IV, 2007, 329-344.

[4] S.M. Han, S.T. Wu, M.A. Dryer, "Three-dimensional, time-dependent numerical modeling of super-sonic, superalfvénic MHD flow”, Comput Fluids, 1988, vol. 16, no.1, pp. 81-103.

[5] P.A. Dawson, "An introduction to magnetohydrodynamics". Cambridge Texts in Applied Mathematics, 2001, ISBN-10: 0-521-79487-0.

[6] M. Abdou, "Introduction to MHD and applications to Thermofluids of fusion blankets": Lecture notes, Institute for Plasma Research (IPR), Gandhinagar, India, 2007.

[7] G.S. Seth, R.N. Jana, M.K. Maiti, "Unsteady hydromagnetic Couette flow in a rotating system". International Journal of Engineering Science, 1982, vol. 20, pp. 989- 999.

[8] T.S. Yusuf, M.O. Oni, "Entropy generation under the influence of radial magnetic field and viscous dissipation of generalized Couette flow in an annulus”, Propulsion and Power Research, 2018, https://doi.org/10.1016/j.jppr.2018.11.005 
[9] S. Das, S.K. Guchhait, R.N. Jana "Radiation effects on unsteady MHD free convective Couette flow of heat generation/absorbing fluid”. Int. J. of Computer Applications, 2012, vol. 39, no. 3, pp. 0975 - 0987

[10] N. Ahmed, "Heat and Mass Transfer in MHD Poiseuille Flow with Porous Walls", Journal of Engineering Physics and Thermophysics, 2019, vol. 92, pp. 122-131. https://doi.org/10.1007/s10891-019-01914W

[11] K. Ilyas, A.S. Nehad, N. Niat, M. Yasir, "MHD mixed convection Poiseuille flow in a porous medium: New trends of Caputo time fractional derivatives in heat transfer problems", The European Physical Journal Plus, 2018, vol. 133, no. 299. https://doi.org/10.1140/epjp/i2018-12105-0

[12] R.M. Gnaneswara, R.K. Venugopal, O.D. Makinde, "Hydromagnetic peristaltic motion of a reacting and radiating couple stress fluid in an inclined asymmetric channel filled with a porous medium”, Alexandria Engineering Journal, 2016, vol. 55, pp.1841-1853.

[13] A. Nayak, G.C. Dash, "Magnetohydrodynamic couple stress fluid flow through a porous medium in a rotating channel", Journal of Engineering Thermophysics, 2015, vol.24, pp. 283-295. https://doi.org/10.1134/S1810232815030091

[14] S.O. Adesanya, B. Souyeh, M. Rahimi-Gorji, M.N. Khan, O.G. Adeyemi. "Heat irreversibiility analysis for a couple stress fluid flow in an inclined channel with isothermal boundaries", Journal of the Taiwan Institute of Chemical Engineers, 2019, vol.101, pp. 251-258

[15] W.N. Mutuku-Njane, O.D. Makinde. "Combined effects of buoyancy force and Navier slip on MHD flow of a Nanofluid over a convectively heated vertical porous plate". The Scientific World Journal. 2013. http://dx.doi.org/10.1155/2013/725643

[16] Y.Lin, L. Zheng, X. Zhang, L. Mad, G. Chen. "MHD pseudoplastic nanofluid unsteady flow and heat transfer in a finite thin film over stretching surface with internal heat generation”. Int. J. Heat Mass Transfer. 2015, vol. 84, pp. 903-911.

[17] S.A. Shehzad, T. Hayat, A. Alsaedi. "Influence of convective heat and mass conditions in MHD flow of nanofluid". Bulletin of the Polish Academy of Sciences Technical sciences. 2015, vol. 63, no.2, pp. 465474. DOI: $10.1515 /$ bpasts-2015-0053

[18] T. Hayat, Z. Abbas, M. Sajid, S. Asghar. "The influence of thermal radiation on MHD flow of a second grade fluid”. International Journal of Heat and Mass Transfer, 2007, vol. 50, pp. 931-941

[19] M. Ahmad, H. Zaman, N. Rehman. "Effects of Hall current on unsteady MHD flows of a second grade fluid". Cent. Eur. J. Phys, 2010, vol. 8, no.3, pp. 422-431.

[20] S.K. Parida, S. Panda, M. Achary, "Magnetohydrodynamic (MHD) flow of a second grade fluid in a channel with porous wall". Meccanica. 2011, vol. 46, no. 5, pp. 1093-1102.

[21] S.O. Adesanya, "Thermal stability analysis of reactive hydromagnetic third-grade fluid through a channel with convective cooling”. Journal of the Nigerian Mathematical Society. 2013; 32: 61-72.

[22] Adesanya S.O, Falade J.A. "Thermodynamics analysis of hydromagnetic third grade fluid flow through a channel filled with porous medium”, Alexandria Engineering Journal. 2015, vol. 57, pp. 615-622.

[23] I. Nayak, A.K. Nayak, S. Padhy, "Unsteady MHD flow and heat transfer of third-grade fluid with variable viscosity between two porous plates". International Journal of Mathematics Trends and Technology (IJMTT). 2018, vol. 54, no. 2, pp. 146-155.

[24] B.I. Olajuwon, J.I. Oahimire, M.A. Waheed, "Convection heat and mass transfer in a hydromagnetic flow of a micropolar fluid over a porous medium”. Theoret. Appl. Mech. Teopm7. 2014, vol. 41, no. 2, pp. 93117, doi:10.2298/TAM1401001B

[25] M.S. Ayano, S.T. Sikwila, S. Shateyi, "MHD Mixed Convection Micropolar Fluid Flow through a Rectangular Duct”. Mathematical Problems in Engineering. 2018, Article ID 9840862, 8 pages, https://doi.org/10.1155/2018/9840862

[26] J.A. Gbadeyan, A.S. Idowu, A.O. Areo, P.O. Olaleye, "The radiative effect on velocity, magnetic and temperature fields of a magnetohydrodynamic oscillatory flow past a limiting surface with variable suction”. Jour. of Mathematical Sciences. 2010, vol. 21, no. 4, pp. 395-411

[27] J.A. Gbadeyan, S. Daniel, E.G. Kefan, "The radiation effect on electrohydrodynamic froth flow in vertical channel”. Journal of Mathematical Association of Nigeria. 2005, vol. 32, pp. 388-396
[28] M. Afranda, D. Toghraieb, A. Karimipoura, W.S. Somchai, "A numerical study of natural convection in a vertical annulus filled with gallium in the presence of magnetic field". Journal of Magnetism and Magnetic Materials. 2017, vol. 430, pp. 22-28.

[29] F. Selimefendigil, H.F. Öztop, "Numerical study of MHD mixed convection in a nanofluid filled lid driven square enclosure with a rotating cylinder". International Journal of Heat and Mass Transfer. 2014, vol. 78, pp. 741-754.

[30] P. Vyas, N. Srivastava, "Entropy Analysis of Generalized MHD Couette Flow Inside a Composite Duct with Asymmetric Convective Cooling”. Arab J Sci Eng. 2015, vol. 40, pp. 603-614.

[31] R.M. Kannan, B. Pullepu, S.A. Shehzad, "Numerical Solutions of Dissipative Natural Convective Flow from a Vertical Cone with Heat Absorption, Generation, MHD and Radiated Surface Heat Flux”. Int. J. Appl. Comput. Math. 2019; 5 https://doi.org/10.1007/s40819-019-0608$\underline{0}$

[32] R. Alizadeh, N. Karimi, A. Nourbakhsh, "Effects of radiation and magnetic field on mixed convection stagnation-point flow over a cylinder in a porous medium under local thermal non-equilibrium”. Journal of Thermal Analysis and Calorimetry. 2019 https://doi.org/10.1007/s10973-019-08415-1(0123456789

[33] M.V. Krishna, G. Reddy, M. Gangadar, A.J. Chamkha. "Heat and Mass Transfer on MHD Rotating Flow of Second Grade Fluid Past an Infinite Vertical Plate Embeddedmi n Uniform Porous Medium with Hall Effects”. Applied Mathematics and Scientific Computing, Trends in Mathematics. 2019. https://doi.org/10.1007/978-3-030-01123-9_41

[34] M.J. Lighthill, "Studies on MHD waves and other anisotropic wave motion”. Phil Trans R Soc, Lond. 1960, vol. 25, no. 2A, pp. 397-430

[35] H. Sato, "The Hall effect in the viscous flow of ionized gas between parallel plates under transverse magnetic field”. J. Phys. Soc. Jpn. 1961, vol. 16, no. 7, pp. 1427-1435

[36] R.S. Nanda, H.K. Mohanty, "Hydromagnetic flow in a rotating channel”. Appl. Sci. Res. 1970, vol. 24, pp. 65-78.

[37] R.N. Jana, N. Datta, B.S. Mazumder, "Magnetohydrodynamic Couette flow and heat transfer in a rotating system”. J. Phys. Soc. Jpn. 1977, vol. 42, pp. 1034-1039.

[38] R.N. Jana, N. Datta, "Hall effects on MHD Couette flow in a rotating system”. Czech. J. Phys. 1980, vol. 330, pp. 659-667.

[39] G.S. Seth, R.N. Jana, M.K. Maity, "Unsteady hydromagnetic Couette flow in a rotating system”. Int. J. Eng. Sci. 1982, vol. 20, pp. 989-999.

[40] A.R. Rao, P.R. Rao, "MHD flow of a second grade fluid in an orthogonal rheometer”. Int J Eng Sci. 1985, vol. 23, no. 12, pp.1387-95

[41] S.R. Kasiviswanathan, M.V. Gandhi, "A class of exact solutions for the magnetohydrodynamic flow of a micropolar fluid”. Int J Eng Sci. 1992, vol. 30, no. 4, pp. 409-417.

[42] A. Bejan, "Entropy Generation Minimization”, CRC Press, Boca Raton, Florida, 1996

[43] S.O. Adesanya, O.D. Makinde. "Thermodynamic analysis for a third grade fluid through a vertical channel with internal heat generation". Journal of Hydrodynamics. 2015, vol. 27, no. 2, pp. 264-272. DOI: 10.1016/S1001-6058(15)60481-4

[44] A.A. Opanuga, O.O. Agboola, J.A. Gbadeyan, H.I. Okagbue. "Entropy generation analysis of Hall current effect on MHD micropolar fluid flow with rotation effect”, SN Applied Sciences, 2020. https://doi.org/10.1007/s42452-019-1783-7

[45] S.O. Adesanya, O.F. Dairo, T.A. Yusuf, A.S. Onaneye, S.A. Arekee, "Thermodynamics analysis for a heated gravity-driven hydromagnetic couple stress film with viscous dissipation effects", Physica A: Statistical Mechanics and its Applications, 2020, vol. 540, pp. 123-150. https://doi.org/10.1016/j.physa.2019.123150

[46] S.O. Adesanya, A.C. Egere, R.S. Lebelo, "Entropy generation analysis for a thin couple stress film flow over an inclined surface with https://doi.org/10.1016/j.physa.2019.121260

[47] A.A. Opanuga, S.O. Adesanya, H.I. Okagbue, O.O. Agboola, "Impact of Hall Current on the Entropy Generation of Radiative MHD Mixed Convection Casson Fluid”, Int. J. Appl. Comput. Math , 2020 https://doi.org/10.1007/s40819-020-0790-0

[48] A.O. Ajibade, B.K. Jha, A. Omame, "Entropy generation under the effect of suction/injection”. Applied Mathematical Modelling. 2011, vol. 35, pp. 4630-4646. 
[49] H. Cao, J. Zhou, J. Song, "Perimeter Entropy and its Application to Climate Change", WSEAS Transactions on Heat and Mass Transfer, 2020, vol. 15, pp. 18-23.

[50] M.I. Khan, T. Hayat, A. Alsaedi, "Numerical investigation for entropy generation in hydromagnetic flow of fluid with variable properties and slip”. Physics of Fluids. 2018, vol. 30. https://doi.org/10.1063/1.5019940

[51] A.A. Sarmad, F.J. Ali, S. Azizan, "Homotopy Perturbation Method Approximate Analytical Solution of Fuzzy Partial Differential Equation”. IAENG International Journal of Applied Mathematics, 2019, vol. 49, no. 1, p. 22-28

[52] M.C. Agarana, M.E. Emetere, "Solving non-linear damped driven simple pendulum with small amplitude using a semi analytical method. ARPN Journal of Engineering and Applied Sciences”, vol. 11, no. 7, pp. 4478-4484.

[53] M.C. Agarana, A.N. Ede, "Application of differential transform method to vibration analysis of damped railway bridge on Pasternak foundation under moving train”. Lecture Notes in Engineering and Computer Science: Proceedings of The World Congress on Engineering and Computer Science. London, U.K. 2016;

[54] O.O. Agboola, J.A. Gbadeyan, A.A. Opanuga, M.C. Agarana, S.A. Bishop, J.G. Oghonyon, "Variational Iteration Method for Natural Frequencies of a Cantilever Beam with Special Attention to the Higher Modes”. Lecture Notes in Engineering and Computer Science: Proceedings of The World Congress on Engineering and Computer Science. London, U.K. 2017;

[55] A.A. Opanuga, E.A. Owoloko, H.I. Okagbue, O.O. Agboola, "Finite difference method and Laplace transform for boundary value problems", Lecture Notes in Engineering and Computer Science: Proceedings of The World Congress on Engineering and Computer Science. London, U.K. 2017;

[56] B. Pullepu, Y. Immanuel, R.M. Selva, NSM Solution on Transient Natural Convective Viscous Fluid Flow Past a Vertical Cone WSEAS Transactions On Heat And Mass Transfer, 2018, vol. 13, pp 1-15.

[57] S.O. Adesanya, J.A. Falade, S. Jangili, O.A. Beg. "Irreversibility analysis for reactive third-grade fluid flow and heat transfer with convective wall cooling”. Alexandria Engineering Journal. 2017, vol. 56, pp.153-160.

[58] A.M. Wazwaz, "Analytical solution for the time-dependent EmdenFowler type of equations by Adomian decomposition method". Applied Mathematics and Computation. 2005, vol. 166, no. 3, pp. 638-651.

[59] A.A. Alderremy, T.M. Elzaki, M. Chamekh, "Modified Adomian Decomposition Method to Solve Generalized Emden-Fowler Systems for Singular IVP”, Mathematical Problems in Engineering, 2019, https://doi.org/10.1155/2019/6097095 